> Au cours de la maladie de Fabry, le déficit en $\alpha$-galactosidase $A$ entraîne une accumulation de globotriaosylcéramide. La quantification et la spéciation de ce lipide peuvent être réalisées par différentes méthodes (en particulier par spectrométrie de masse en tandem couplée à une technique séparative) qui sont décrites et analysées. <

\section{Quantification et spéciation du globotriaosylcéramide}

Sandrine Roy, Dominique P. Germain, Arlette Baillet, Patrice Prognon, Pierre Chaminade

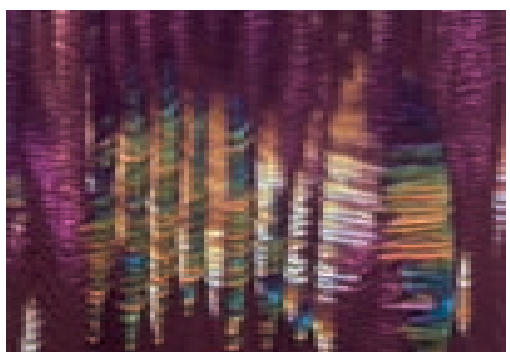

S. Roy A. Baillet, P. Prognon, P. Chaminade: Groupe de Chimie Analytique du Sud de Paris, $\varepsilon A$ 3343, Faculté de Pharmacie de Paris XI, 5, rue Jen-Baptiste Clément, 92296 ChâtenayMalabry Cedex, France. D.P. Germain : Unité Fonctionnelle de Génétique Clinique. Hôpital Européen Georges Pompidou, 20, rue Leblanc, 75015 Paris, France.

pierre.chaminade@cep.u-psud.fr

Le globotriaosylcéramide $\left(\mathrm{Gb}_{3}, \mathrm{GL}-3\right.$ ou CTH) est un glycosphingolipide ubiquitaire de l'organisme. II est constitué d'un trihexoside, polaire, et d'un squelette céramide, apolaire. La partie osidique, de structure fixe, implique que le $\mathrm{Gb}_{3}$ constitue une classe lipidique. Au sein de cette classe, les variations structurales du squelette céramide (longueur des chaînes hydrocarbonées, nombre et positions de doubles liaisons et/ou de groupements hydroxylés) permettent, quant à elles, de définir la notion d'espèces moléculaires, ou isoformes, au sein de la classe lipidique.

Au cours de la maladie de Fabry, l' $\alpha$-galactosidase $A$, rendue non fonctionnelle suite à une mutation du gène $G L A$, n'est plus à même d'hydrolyser le $G_{3}$, ce qui conduit à son accumulation dans le plasma, les tissus et l'urine. La quantification de ce lipide et l'étude de ses variations structurales impliquent dans un premier temps de l'isoler au sein des échantillons biologiques étudiés. Par la suite, l'étude de ce glycosphingolipide revêtira deux aspects : d'une part, l'analyse de la classe dans son ensemble dans un but diagnostic ou quantitatif, d'autre part, la détermination des différentes structures en présence.

Différentes approches ont été proposées dans la littérature pour procéder à l'analyse de la classe lipidique. II s'agit notamment de techniques immunologiques, comme la technique ELISA (enzyme linked immunosorbent assay), ou de chromatographie liquide de phases normales. Si les principes de ces techniques peuvent paraître dissemblables, elles possèdent néanmoins un point commun, dans le sens où elles s'adressent à la partie invariable de la molécule, le trihexoside.

La technique ELISA [1] est originale dans la mesure où la détection $\mathrm{du} \mathrm{Gb}_{3}$ n'est pas assurée par la mise en œuvre d'un anticorps anti-Gb 3 , mais par la sous-unité $B$ de la vérotoxine (VTB) d'Escherichia coli, qui présente une affinité importante pour le glycosphingolipide considéré. Le complexe $\mathrm{Gb}_{3}-\mathrm{VTB}$ est, ensuite, révélé par un anticorps anti-VTB. Si cette technique présente l'avantage d'être automatisable et donc de traiter rapidement un grand nombre d'échantillons, le risque de réactions croisées reste important.

La technique de chromatographie liquide repose, quant à elle, sur l'affinité qui se développe entre trois parties: le soluté analysé, la phase stationnaire, sur laquelle le soluté peut s'adsorber, et la phase mobile qui permet l'élution du soluté. En optant pour une phase stationnaire polaire, les interactions phase stationnaire-soluté dépendent de la polarité du soluté, et donc de l'importance de la chaîne osidique. Cela permet de séparer les différents glycosphingolipides physiologiques [2], et notamment le $\mathrm{Gb}_{3}$ et le galabiosylcéramide $\left(\mathrm{Ga}_{2}\right)$, second glycosphingolipide neutre s'accumulant au cours de la maladie de Fabry. La Figure 1 illustre le chromatogramme obtenu suite à l'analyse du $\mathrm{Gb}_{3}$ concentré dans les sédiments urinaires d'un enfant affecté par la maladie de Fabry. Si cette technique nécessite d'analyser successivement les différents échantillons, la spécificité apportée par la séparation chromatographique permet de réduire considérablement le risque de réactions croisées et donc de faux-positifs. 
Si ces deux techniques permettent de mettre en évidence un excès de $\mathrm{Gb}_{3}$, la structure des espèces moléculaires reste inaccessible. Seule la mise en œuvre de techniques de spectrométrie de masse donne accès à des renseignements structuraux. Quelles que soient les techniques utilisées, elles consistent à analyser des solutés sous forme ionisée. Si l'Electrospray [3] reste le mode d'ionisation le plus décrit, le MALDI (matrix assisted laser desorption ionisation) [4] et plus récemment I'APPI (atmospheric pressure photo-ionisation) [5] permettent, de la même manière une ionisation des molécules étudiées. L'ionisation s'effectuant dans des conditions douces, les solutés seront ionisés sans avoir été fragmentés, ce qui permettra d'accéder à la masse moléculaire, mais reste insuffisant pour distinguer des espèces isobariques (espèces présentant une même masse moléculaire mais une structure différente).

Pour différencier ces espèces isobares, deux approches sont possibles: la spectrométrie de masse tandem (ou MS/MS) ou la spectrométrie de masse couplée à une technique séparative, comme la chromatographie liquide. Le principe de la MS/MS consiste à sélectionner un ion parmi l'ensemble des ions formés et à le fragmenter. L'analyse des fragments obtenus permettra alors de déterminer la structure de la molécule. La sélection de l'ion qui sera fragmenté s'effectue par sa masse moléculaire. En présence d'espèces isobares, les fragments obtenus seront différents et ainsi, ces différentes espèces moléculaires seront mises en évidence. Dans la seconde approche (couplage à une technique séparative), les différentes espèces en présence sont séparées dans un premier temps, puis sont analysées sous forme ionisée. La technique séparative la plus souvent utilisée est la chromatographie liquide. Dans ce cas, puisque l'on cherche à séparer les différentes isoformes portées par la partie apolaire $d u \mathrm{~Gb}_{3}$, une phase stationnaire apolaire sera utilisée pour générer des interactions avec le squelette céramide. En présence d'une technique séparative hautement résolutive, les espèces isobares seront séparées, mais leurs structures resteront indéterminées. C'est pourquoi, les techniques de chromatographie sont de plus en plus couplées à des techniques de MS/MS qui donneront accès à des données structurales.

Si les trois techniques d'ionisation précédemment évoquées permettent de procéder aux analyses de structure, l'ajout d'un étalon interne (composé initialement absent de l'échantillon et ajouté en quantité connue à cet échantillon) a contribué à l'essor de la quantification du $\mathrm{Gb}_{3}$ par spectrométrie de masse.

Il apparaît donc que l'analyse du globotriaosylcéramide est complexe, que soient considérés ses aspects quantitatifs ou qualitatifs. Le développement de la spectrométrie de masse a constitué un apport important du fait de la sensibilité et de la spécificité qui caractérisent cette technique d'analyse. $\diamond$

\section{SUMMARY}

Quantification and speciation of globotriaosylceramide During the course of Fabry disease, the deficiency in alpha galactosidase $A$ results in accumulation of globotriaosylceramide. Quantification and speciation of this lipid may be performed by several technics (particularly by coupling separative techniques with tandem mass spectrometry) which are described and analysed. $\diamond$

\section{RÉFÉRENCES}

1. Zeidner KM, Desnick RJ, loannou YA. Quantitative determination of globotriaosylceramide by immunodetection of glycolipid-bound recombinant verotoxin B subunit. Anal Biochem 1999; 267 : 104-13.

2. Roy S, Gaudin K, Germain DP, et al. Optimisation of the separation of four major neutral glycosphingolipids : application to a rapid and simple detection of urinary globotriaosylceramide in Fabry disease. J Chromatogr B 2004 ; 805 : 331-7.

3. Mills K., Johnson A, Winchester B. Synthesis of novel internal standards for the quantitative determination of plasma ceramide trihexoside in Fabry disease by tandem mass spectrometry. FEBS Lett 2002 ; 515 : 171-6.

4. Touboul D, Roy S, Germain DP, et al. Fast fingerprinting of urinary sediment glycosphingolipids by MALDI-TOF mass spectrometry in Fabry disease. Anal Bioanal Chem 2005 ; 382 : 1209-16.

5. Roy S, Delobel A, Gaudin K, et al. Porous graphitic carbon with atmospheric pressure photo-ionisation mass spectrometry and tandem mass spectrometry as coupling tools for the analysis of glycosphingolipids molecular species. J Chromatogr A 2005 (sous presse).

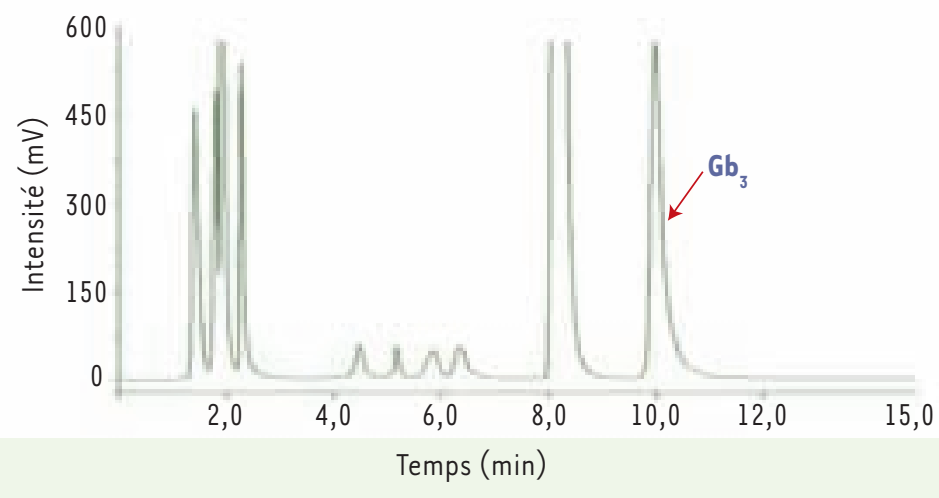

Figure 1. Analyse chromatographique du sédiment urinaire obtenu à partir d'un échantillon de $20 \mathrm{ml}$ d'urines d'un hémizygote atteint de maladie de Fabry [2]. 